\title{
Vitamina E
}

Dra. Mireya Bravo*, Q.F. Olga Puratić, Dr. Renê Stephan* y señorita Iris Oviedo (T.M.)*

\section{ANTECEDENTES}

En 1922, Evans y Bishop reconocieron la existencia de un factor esencial para la reprodución en la dieta de animales. Este factor fue denominado Vitamina $E$, se le llamó además tocoferol (tocos: nacimiento, phero: traer), debido a sus propiedades antiestériles en la rata ${ }^{1-2-3}$.

Aun cuando desde 1937 se conocen sus propiedades antioxidantes, la investigación estuvo relacionada fundamentalmente a los órganos y funciones de la reproducción, y sólo en los úlmos años ha empezado a tomar importancia el est udio de su función en la nutrición humana'.

Se han descrito varios sindromes por deficiencia, tanto en animales como en el hombre; el exceso de Vitamina $E$, es un tema en estudio actualmente $y$ aun cuando no parece tener efectos nocivos a corto plazo, no se conocen sus efectos a largo plazo ${ }^{156}$.

\section{CARACTER1STICAS QUIMICAS DE LA VITAMINA E}

El tocoferol es el compuesto principal de esta vitamina, corresponde químicamente a un

\footnotetext{
-Departamento de Pediatria, Hospital Roberto del Rio, Santiago.
}

derivado metil-6-hidroxicromano, con una cadena alifática de 16 carbones laterales.

El compuesto natural más activo es el d-alfatocoferol, pero existen en forma natural diversos compuestos dependientes de la posición del metilo en el anillo cromano y de la cadena lateral en $\mathrm{C}_{2}$, esta última responsable de sus propiedades liposolubles.

Asi hay 4 formas de tocoferoles $(\alpha, \alpha, \gamma$,

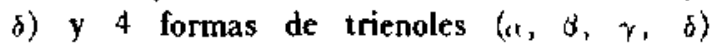
(Fig. 1) que tienen diferente absorción intestinal y retención en los diversos tejidos, y, por lo tanto, distinta actividad biológica ${ }^{1-7}$

Estos compuestos son relativamente inestables expuestos al aire, por lo que las formas comenciales son en su mayoría ésteres de acetato o succinato, to que las hace estables en diversas condiciones.

La estandarización internacional se hizo con la forma sintética de acetato de d-l-alla-tocoferol, y $1 \mathrm{mg}$ corresponde a 1 Unidad Internacional (UI) ${ }^{1}$ (Fig 1).

La vitamina $\mathbf{E}$ se encuentra ampliamente distribuida en los alimentos, de éstos las grasas y aceites contienen más de la mitad de la Vitamina E de la dieta. Otras fuentes son los cereales; aves, carnes, pescados, etc. El contenido de la leche humana es de 0,2 U.I. por $100 \mathrm{Kcal}$, y el 


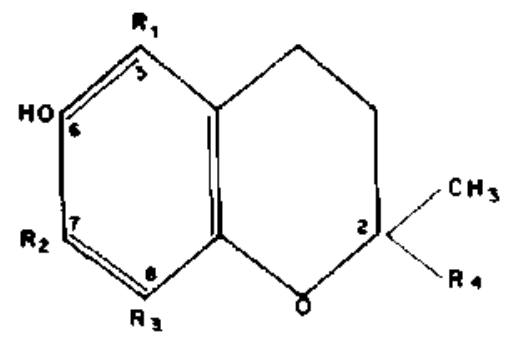

TOCOFEROLES

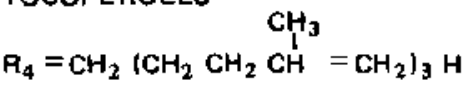

TRIENOLES

$\mathrm{R}_{4}=\mathrm{CH}_{2}\left(\mathrm{CH}_{2} \mathrm{CH} \stackrel{\mathrm{C}}{\mathrm{C}} \mathrm{C}=\mathrm{CH}_{2}\right)_{3} \mathrm{H}$

Fig. 1

Formas de tocoferoles naturales.

de la leche de vaca 0,1 U.I. por $100 \mathrm{Kcal}$. Las leches industrializadas tienen contenidos variables de Vitamina $\mathbf{E}$.

\section{REQUERIMIENTOS}

Los requerimientos de Vitamina $\mathrm{E}$ tanto del niño como del adulto no están claramente establecidos, ya que para determinarlos, es necesario tomar en cuenta diversos factores, algunos de los cuales son dificiles de evaluar. Estos factores son:

- La síntesis e incorporación de ácidos grasos poliinsaturados (P.U.F.A.) por el organismo.

- El porcentaje de P.U.F.A. de la dieta.

- El consumo de P.U.F.A. por el organismo, ya sea por depósito en la célula u otra forma de metabolización.

Aun cuando se han hecho diversas recomendaciones en relación a esta vitamina, en este momento el requerimiento para el adulto está calculado entre 10 a 30 U.I. por dia ${ }^{1-4}$, dependiendo del contenido de P.U.F.A. en la dieta. En el prematuro, los requerimientos son altos debido a que los depósitos y la absorción son bajos, y dependen del contenido de P.U.F.A. de la leche que se administra. El requerimiento para niños de menos de $1.500 \mathrm{~g}$ se ha calculado entre 5 a 25 U.I. por dia, durante los 3 primeros meses de vida y para niños de término de 0 a 1 año en 1 U.I. por $100 \mathrm{Kcal}^{\mathrm{g}}$.

\section{ABSORCION INTESTINAL}

La absorción intestinal de Vitamina $\mathrm{E}$ es relativamente baja y es del orden del $20-30 \%$. En el niño de bajo peso de nacimiento la absorción es auún menor.

\section{NIVELES SANGUINEOS}

Los niveles séricos de d-alfa-tocoferol pueden no reflejar ni la ingesta ni los depósitos. El tocoferol se asocia a las lipoproteinas y se distribuye de acuerdo al contenido de grasa de cada fracción. Parece ser que los valores de tocoferol séricos pueden tener poco valor si no se acompañan de los valores de lípidos plasmáticos. Se ha sugerido que una relación de $0,8 \mathrm{mg}$ de tocoferol total por gramo de lípidos plasmáticos totales podría indicar un buen estado nutricional a este respecto ${ }^{4}$.

El test de hemólisis de glóbulos rojos por peróxido de hidrógeno es un buen indicador de los niveles de tocoferol plasmáticos. Asi se ha visto que hay una correlación indirecta entre niveles plasmáticos de tocoferol y porcentaje de hemólisis, especialmente cuando esta última es alta, reflejando con alta probabilidad una deficiencia de Vitamina $E^{\prime}$. Debido a la facilidad con que puede realizarse este test en nuestro medio, es de gran utilidad en la práctica clíni$\mathrm{ca}^{10-11}$.

\section{FUNCION DE LA VITAMINA E}

La Vitamina $\mathbf{E}$ tiene diversas funciones metabólicas. Entre ellas tal vez el más importante y mejor estudiado es el papel protector de las membranas biológicas, ya sea evitando la oxidación de sus componentes celulares esenciales o evitando la formación de productos tóxicos de oxidación como los peróxidos de ácidos grasos no saturados, actuando así como estabilizador de la estructura lipídica de los tejidos ${ }^{1-4-d-12-13 \ldots 14}$.

Bien conocidas son sus funciones en relación a la reproducción ${ }^{2}$. Se ha relacionado además la carencia de Vitamina $\mathbf{E}$ con alteraciones en el sistema muscular y cardiovascular especialmente en animales ${ }^{14}$. Por otro lado, se le han reconocido efectos terapéuticos en la fibroplasia retrolental ${ }^{15}$ y en la claudicación intermitente ${ }^{18}$. En los últimos años se han estudiado atras funciones de la Vitamina $\mathbf{E}$, corno por ejem- 
plo un papel en la síntesis del Hem a través de la sintetasa del ácido delta-amino-levulinico en la médula ósea; ésta disminuiría en casos de carencia de Vitamina E. . .

Otros sistemas enzimáticos estarian también alterados en relación a esta carencia; por ejemplo, se ha encontrado que aumenta la actividad de la xantino-oxidasa hepática. Sin embargo, no hay aún resultados conchuyentes $y$ queda por demostrarse que una reacción enzimática determinada tiene un requerimiento especifico de d-alfa-tocolerol ${ }^{14}$.

También se ha reconocido últimamente, efectos protectores de la Vitamina $\mathrm{E}$ a la acción de algunos tóxicos químicos. En este sentido, hay grupos de trabajo estudiando la prevención por la Vitamina $E$ de los efertos tóxicos de la contaminación atmosférica' ${ }^{1}$.

De gran importancia son los estudios en relación a Hipervitaminosis $\mathrm{E}$ y coagulación. Parece haber una interferencia directa entre actividad de la Vitamina $E$ y $K$, por lo que se ha descrito, asociado a grandes dosis de Vitamina $\mathrm{E}$, aumento del tiempo de protrombina y disminución de los factores dependientes de la vitamina $K$, lo que ha sido utilizado en el tratamiento de enfermedades cardiovasculares ${ }^{5}$.

Por ot ra parte, se ha visto experimentalmente que productos de oxidación de la Vitamina $\mathrm{E}$ como d-alfa-locoferil hidroquinasa, curan la distrofia muscular nutricional en animales ${ }^{5}$

\section{CARENGIA DE VITAMINA EN}

\section{EL PREMATURO}

Desde que Oski, en $1967^{3}$ describió una forma de anemia hemolítica en el prematuro en relación con la carencia de Vitamina $\mathrm{E}$, numerosos autores han estudiado y comprobado esta relación que en un comienzo fue mirada con mucho escepticismo.

Actualmente el cuadro clínico y de laboratorio está definido, aun cuando se presenta en un período en que las anemias son de difícil interpretación ${ }^{8 \cdot 17 \cdot 18} 19$.

El recién nacido tiene niveles bajos de tocoferol, y en el niño de bajo peso de nacimiento estos son aún menores ${ }^{13-20 \cdot 21}$. Estos niveles bajos resultan de la combinación de diversos factores:

1. Depósitos bajos al nacer. Debido a que el contenido de tocoferol del recièn nacido, y en especial del niño de bajo peso es muy bajo, y éste no tiene relación con los valores maternos, se ha sugerido que se deba a una alteración en el transporte de Vitamina $\mathbf{E}$ a través de la placen$\mathbf{t a}^{20}$. Al administrar Vitamina $\mathrm{E}$ a la madre, obteniendo niveles séricos altos en éstas, no se ha logrado un aumento adecuado de niveles de Vitamina E en el cordón.

Los depósitos bajos persisten durante las primeras semanas de vida y son especialmente bajos en el niño de bajo peso de nacimiento. Asi el recién nacido de $1.000 \mathrm{~g}$ entra al periodo de "anemia fisiológica" con depósito de 3 mg en vez de $20 \mathrm{mg}$ de los niños de $3.500 \mathrm{~g}^{8}$.

2. Aporte insuficiente. El aporte de Vitamina $E$ de las leches es, en general, bajo, pero ya que los requerimientos de Vitamina $\mathbf{E}$ están en relación directa con el contenido de P.U.F.A., es importante relacionar estos valores. Así se ha expresado esta relación entre mg de alfa tocoferol con gramo de P.U.F.A. de la dieta. Se ha calculado que la relación E/P.L.F.A., que previene la carencia, es aproximadamente de 0,6. Las leches artificiales con agregados de aceites vegetales tienen un alto contenido de P.U.F.A. y la relación E/P.U.F.A. es baja. Los niños alimentados con estas leches tienen por lo tanto niveles aún más bajos de Vitamina $\mathrm{E}$ en relación a las necesidades. Los niños alimentados con leche materna, cuya relación E/P.U.F.A. es de 1,0 , tienen niveles normales de tocoferol ${ }^{22}$.

La composición de ácidos grasos de la dieta se refleja en la composición de ácidos grasos del tejido adiposo y de la membrana del glóbulo rojo ${ }^{20-23}$. Habiendo una relación directa entre el contenido de P.U.F.A. de la dieta y en la membrana del glóbulo rojo ${ }^{24}$. Este hecho es muy importante de tener presente para explicar el mecanismo de la hemólisis en la carencia de Vitamina $\mathbf{E}$.

3. Absurción intestnal. En el niño de bajo peso hay una mala absonción en Vitamina $\mathbf{E}$ durante las primeras semanas de vida, especialmente cuando se administran dietas con bajo contenido graso ${ }^{13}{ }^{25 \cdot 26}$. Melhorn y Gross ${ }^{27}$ demostraton que hay una relación directa entre edad gestacional y niveles de Vitamina $\mathbf{E}$ alcanzados en las primeras semanas de vida. La mala absorción mejora a medida que se alcanza la edad cronológica equivalente al recién nacido de término. Se ha visto que aun suplementando por vía oral con Vitamina $E$, en dosis equivalen- 
tes a los requerimientos del adulto (25 U.l. de acetato de tocoferol), no se logran obtener niveles séricos normales de Vitamina $E$. Es por esta razón que las dosis terapéuticas son extraordinariamente altas en relación a los requerimientos.

En el recién nacido de término la absorción es buena y los niveles séricos están en relación con los aportes de la dieta ${ }^{20}$.

Es importante tener presente que en la absorción de Vitamina $\mathrm{E}$ influyen otros factores, como es la presencia en el intest ino de otmos nutrientes y en especial del fierro. Este último disminuye la cantidad de alta tocoferol disponible para absorción por efecto oxidante sobre la vitamina liposoluble ${ }^{22-28}$. La mala absorción de la $\mathrm{Vi}$ tamina $E$ parece estar relacionada además con la falla de absonción de grasas de los niños de bajo peso ${ }^{20}$.

Gross y Melhorn compararon resultados de niveles séricos de Vitamina $\mathrm{E}$ y respuesta hematológica en recién nacidos a los que se suplementó Vitamina $\mathbf{E}$ en forma liposoluble e hidrosoluble, $y$ vieron que se obtenian niveles más altos de tocoferol y hemoglobina cuando se usaba la forma hidrosoluble ${ }^{30}$.

\section{RELACION ENTRE VITAMINA E Y ANEMIA HEMOLITICA}

Hemos visto que la composición de grasas de la dieta se refleja no sólo en la composición del tejido adiposo sino también de la membrana celuiar y célula misma. En la composición de la membrana celular, esto es especialmente cvidente en relación al contenido de ácidos grasos no saturados.

Los ácidos grasos no sáturados son fácilmente oxidable. Para evitar esta oxidación y mantener por lo tanto la estructura, necesitan de la presencia de antioxidantes a nivel telular comon es la Vitamina F.".

Cuando hay deficiencia de Vilamina $\mathrm{E}, \mathrm{la}$ membrana del glóbulo rojo es alteradi por prroxidación de lípidos. lous que so atcun lan ? is unen a grupos sulfidrios. Estä alteracion de la membrana del globulo rojo, peranite que este se haga hiperpermeable a cationes, pronluciénduse un aumento de volumen osmotico seguirto de hemolisis, que se traduce en acoramento de be

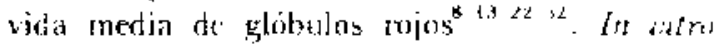

cs posible corregir esta hemólisis con el agregado de tocoferol ${ }^{1 !}$.

\section{INTERACCION ENTRE FIERROY VITAMINA E}

Se ha visto in vivo que el fierro disminuye la absorción intestinal de Vitamina $E$, por otra parte in vitro, cuando se encuentra libre, actúa como cofactor catalizando la ruptura oxidativa de los ácidos grasos no saturados de la membrana del glóbulo rojo. Presumiblemente grandes dosis de fierro in vivo tienen igual efecto ${ }^{83}$.

Los trabajos de Melhorn y Gross" han demostrado claramente que al administrạr fierro a prematuros durante las primeras semanas de vida, éstos tienen un grado mayor de anemia y hemólisis que aquéllos a los que se administra Vitamina E, Vitamina $\mathrm{E}$ más fjerro, o ningún suplemento. Igual experieлcia se encuentra en los trabajos de M. Williams ${ }^{34}$.

Por esto se ha sugerido que ya que en los depósitos de fierno no se han depletado en el prematuro antes de los 2.3 meses de vida, sería mejor no iniciar en ellos la suplementación con fierro antes de los 3 meses de edad" ${ }^{34}$.

\section{CUADRO CLINICO}

La anemia hemolítica por carencia de Vitamina $E$ se presenta exclusivamente en nisios de bajo peso de nacimiento con alimentación artificial. Es un problema autolimitado del primer trimestre de la vidá y se manifiesta a partir de la 4-6 semana.

Clínicamente se encuentran niños pálidos, que pueden presentar edema de extremidades inferiores, párpados, genitales. Se ha descrito además rinorrea acuosa, taquipnea $e$ inquietud.

Desde el punto de vista hematológico, se calracteriza por valores de hemoglobina bajo to esperado para la edad y peso de nacimientu, in general en un rango de 7 a 11) g, con aumento ditl número de reticulocilos, sugiricndo el proceso hemolitico. Este hecho es imporiante en el tritnejo chiniro del enfermo ya que por imalarse de as periodo de "anemia fisiolingica" a menude este aumenuc de reticulowios es mal interpretado.

Mordologicamente se carartertza por aniso y poiquilocitosis. fragmentación de glóbulos rwjos, algunos esterixitos y policronuatófilos. 


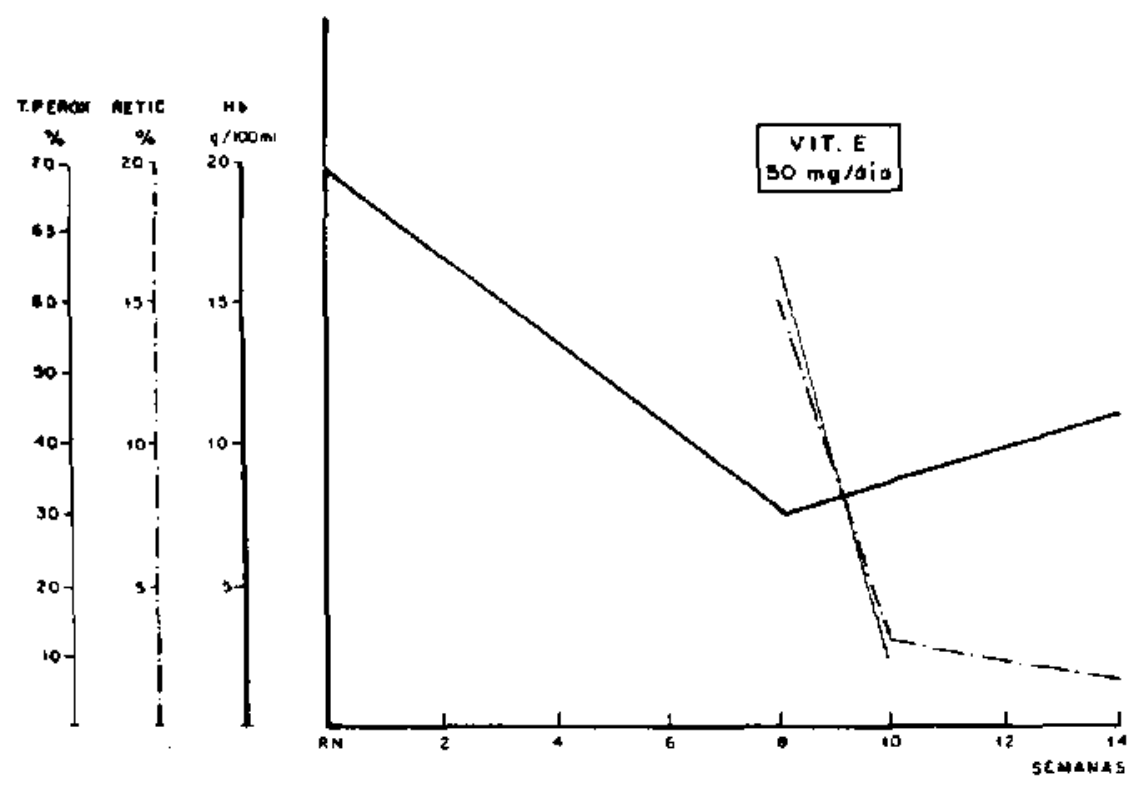

Fig. 2

Evolución hematológica de un R.N.P.T. A.E.G., P.N.: $900 \mathrm{grs.,} \mathrm{trafado} \mathrm{con} \mathrm{vitamina} \mathrm{E}$

El aumento del número de plaquetas es un hecho frecuente ${ }^{23-34}$ que ha sido encontrado incluso en forma experimental en ratas carentes de Vitamina $E^{25}$.

En la médula ósea se observa hiperplasia eritroide sin alteraciones megaloblásticas. Se han descrito algunos precursores eritroides poliploides y cambios en el pattern de cromatina, con depósitos de ella en la periferia del núcleo con un área central pálida. Estos cambios son similares a los observados en monos carentes de Vitamina E. $^{26}$

Se ha demostrado que la sobrevida de glóbulos rojos, medida con $\mathrm{Cr}_{31}$ está disminuida en enfermos con carencia de Vitamina $E^{22} 35$. El tocoferol sérico está disminuido y hay aumento de la hemólisis medida con el test de Peróxido de Hidrógeno ${ }^{9-10-11-16}$. Estas alteraciones de laboratorio se han corregido con el tratamiento con Vitamina $\mathrm{E}^{3-13}$

Por otra parte, hemos seguido la evolución hematológica de 18 recién nacidos de menos de $2.500 \mathrm{~g}$, hasta las 12 semanas de edad; en 6 encontramos signes de hemólisis entre la $6^{\circ}$ a $8^{4}$ semana de vida. Las características de laboratorio están resumidas en la tabla 1. Clinicamente sólo encontramos palidez franca en 4 , edema de extremidades en 3 y rinorrea en 1.
Tabla I

\begin{tabular}{|c|c|c|}
\hline & Promerfio & Rong'í \\
\hline Peso de nacimientog. & 1946 & $1150 \cdot 2200$ \\
\hline Hemoglobina $\mathrm{g} / 1 \mathrm{c}(\mathrm{l} \mathrm{ml}$. & 8.1 & $5.8-9.5$ \\
\hline Hematorrito \% & 23.7 & $17.5-27.0$ \\
\hline Retirulocitos \% & 62 & $3.1 \cdot 10.6$ \\
\hline Plaquetas $\times \mathrm{mm}$ & 42006 & $3(\mathrm{~N}) 000)-706000$ \\
\hline Test henoúlisis por $\mathrm{H}_{2} \mathrm{O}_{2} \%_{\%}$ & 60.90 & $46.32-84.50$ \\
\hline
\end{tabular}

Características te laboratorio de ó prematuros que presentaran anemia hemolicica por carencia de vitamina "E" entre la 6 y y 8 " semana de vida.

\section{TRATAMIENTO}

Como ya hemos dicho, la anemia hemolítica por carencia de Vitamina E es un problema autolimitado del niño de bajo peso en los primeros 3 meses de vida, y se debe no sólo a las caracteristicas propias del niño prematuro (depósitos bajos, absorción pobre, etc.) sino está relacionada además con factores de la dieta como: contenido de ácidos grasos poliinsaturados y Fe. Por lo tanto, deberán tenerse presente en la prescripción dietética del niño de bajo peso en las primeras semanas de vida. 
En cuanto a la administración de Vitamina $E$, hay que distinguir la administración como suplemento, que debería hacerse a todo niño de bajo peso con alimentación artificial, del tratamiento de la carencia.

Como suplemento se han sugerido diversas dosis que van entre 5 a 25 U.I. de acetato de alfa tocoferol, en forma oral diaria, a partir de los 10 dias de vida y hasta el tercer mes ${ }^{2}{ }^{3}$. La mayor parte de los autores parecen estar de acuerdo que dosis de 10 U.J. serian suficientes para prevenir esta carencia ${ }^{\mathbf{B}}$.

En cuanto al tratamiento de la carencia, tanto la dosis como el período durante el cual debe administrarse han sido variables y discutidos. Sin embargo, parece ser que $100 \mathrm{U}$.l. de acetato de alfa tocoferol por via oral, divididas en 4 dosis, lejos de la alimentáción, serían suficientes. El componente hemolitico desaparece o disminuye francamente 4 días después de iniciado el tratamiento. La duración de éste ha sido variable entre 4 dias y meses ${ }^{22}$. Sin embargo, en la práctica hemos visto que han sido útiles tratamiento entre 7 a 25 dias. La experiencia con Vitamina $E$ hidrosoluble ha sido buena, $y$ seguramente las dosis a usar, cuando esté disponible comercialmente, serán menores ${ }^{30}$.

El uso intramuscular se recomienda hacerlo en dosis no mayores de $50 \mathrm{U}$.l. y administrarlo no más de dos veces a la semana

\section{BIBLIOYRAFIA}

1 Horatt W. K. Am. J. Cilin. Nutr. 20.369, 1976.

* Bifeer. R.; Coldstetn, B.l). Seminars in Hematology, January, 1970.

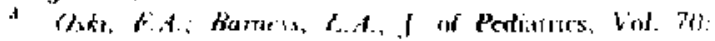
$211,196 \%$

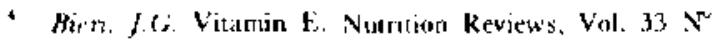
6. $161,19 ? \%$

5 Ilipervitaminosis $F$ and tagulation. Nutrition Revirus, vid. 3 is $\mathrm{N}^{\mathrm{a}}$ !, 1075

b Hurush. H.A. International simposium an Vitamin E, 1974

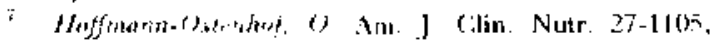
147.

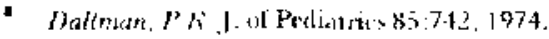

- Leonard, P.J.; Loswsky, M.S. Am. J. Clin. Nutr. 20: 8, 795, 1967 .

14 Melhom. D.K.; Gross, S; Irak, G.A. and Leu, J.A. Blood, 37:438, 19? I

1 Catherine, S. Rose and Paul Cyörgy. Am. J. of Physiology, $168: 414,1952$.

12 Vitamin $\mathrm{E}$ therapy in prenature babies. Nutrition Reviews, 33: 7, 1975.

13 Lo Sis,; Fronk I.; Hitzi, W.H. Arch. Dis. Child., 48 360,1973

14 Grodman, L.S. and Gilman, A. The pharmacological hasis of therapeutics, $5^{\circ}$ Ed., Macmillan Publishing Co., 1975.

is Johnsan, L. D.; Sichaffer, D.; Biggs, TR. Am. J. Clin. Nutr., 27:1158, 1974

10 Haeger, K. Am. J. Clin. Nutr. 27:1179, 1974.

17 Stockstan, J.A. Seminars in Hematology, $12: 2$. 163,1975

18 Osk, F.A. British Journal of Harmatology, 27: 195,1974

19 Schutman. I. J. Pediat., 54:663, 1959

- Vobecky, J.S.; Vobecky. J.; Shapcott, D., Blanchard, $R$ Am. J. Clin. Nutr, 29:776, 1976.

21 Hassan, H.; Hashm, S.A.; Van hallie, T.B. and Sebrell, W.H. Am. J. Clin. Nur., 19:147, 1966.

${ }^{22}$ Ritchie, J.H.; Fish, M.B.; Mc Masters, $V^{\prime}$ and Gronsmann, M. New Engl. J. of Med., 279:1185, 1968.

a Witting. L.A. Intemational Symposium on Vitamin E. 1974

24 Brin, M.; Hom, L.R.; Barker, M.O. Intemational Symposium on Vitamin $E, 1974$.

oski, F.A. and Bamess, J.A. Am. J. Clin. Nulr, 21 45,1968 .

26 Vitousky, H.M.; Comblath, M.; and Ginfor, II.II Am. J. Dis. Child., 92:164, 1956.

"Melhom, D.K.; Gross, S, and Childers, (;. J- of Pedial. $79: 5 \times 1,1971$.

at Gimss, S. and Methom, D.K. Ann, $\mathbf{N}$ Y Arsd. iro. $203: 141.1972$.

to Katz, Lame; Hamilion, J.R. J. of Pediat., 95:\{, 608, 1479

st Gross, S.; Methom, D.K. J. of Pedial, 85:6, "53, 1974

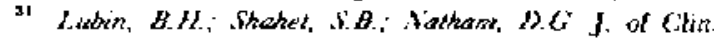
Invest., $51: 338,1072$.

32 Honsitl. MK.; Century, B.; Zeman. A.A. Am. J. Cilin. Nutr , 12.99, 1963.

9 Methom. D.K.; Gross, 5 . The journal of Pediatrics. $79 \cdot 4,569,1971$

34 Writhams, M.l.; Shatt, R.f.; O'Neat, P.L.; Oski, F.A New. Engl. J. of Med. 292:17, 887, 1975.

is Binder, H.J; Hertig, D.C.; Hurst, V.; Finch, S. and Spiro, H.M. N. Engt J. ol Med . 273:1289, 1965. 